\title{
Eficiência agrícola da produção de soja, milho e trigo no estado do Rio Grande do Sul entre 1980 e 2008
}

\author{
Agricultural efficiency of soybean, corn and wheat production in the state of Rio Grande do Sul, \\ Brazil, between 1980 and 2008
}

\author{
Rafael Battisti $^{{ }^{*}}$ Paulo César Sentelhas $^{\mathrm{I}}$ Felipe Gustavo Pilau $^{\mathrm{II}}$
}

\section{RESUMO}

\begin{abstract}
Eficiência agrícola (EA) é utilizada como indicador do nível de desenvolvimento agrícola regional, expressando, por meio da relação entre as produtividades real e atingível, o nível tecnológico empregado nas culturas. Com base nisso, o objetivo deste estudo foi avaliar a EA das culturas da soja, do milho e do trigo para o estado do Rio Grande do Sul, entre os anos de 1980 e 2008, identificando os principais fatores que as condicionaram. A EA foi obtida pela relação entre a produtividade atingivel (PA) e a real (PR). A PR foi obtida junto ao banco de dados do Instituto Brasileiro de Geografia e Estatística (IBGE). A PA foi obtida pela estimativa da produtividade potencial (PP), pelo método de Zona Agroecológica da FAO, deflaciónada pelo déficit hídrico em cada uma das fases da cultura. Verificou-se que as EAs médias para as culturas do milho, da soja e do trigo para o RS foram iguais a 54, 61 e 43\%, respectivamente. Nas localidades de Santa Rosa, São Borja e Veranópolis, a EA para a soja foi, ao contrário das demais localidades, negativa. Os principais fatores que contribuíram para o aumento da EA, na maioria das localidades, foram: mudanças no uso e fertilidade do solo; uso de mecanização agrícola; preços pagos pelas commodities; investimentos em pesquisa e desenvolvimento; adoção do zoneamento de risco climático; e melhoramento genético.
\end{abstract}

Palavras-chave: evolução tecnológica, produtividade potencial, produtividade atingível $e$ produtividade real.

\section{ABSTRACT}

Agricultural efficiency (EA) is used as an indicator of the level of regional agricultural development, reflecting, by mean of the ratio between actual and achievable yields, the

\begin{abstract}
crop technology level. Based on that, the objective of this study was to evaluate the EA for soybean, corn and wheat crops in the state of Rio Grande do Sul, Brazil, between 1980 and 2008, identifying the main factors which conditioned it. EA was obtained by the relationship between the achievable yield (PA) and actual crop yield (PR). PR was obtained from the Brazilian Institute of Geography and Statistics (IBGE) data base. PA was obtained by estimating the potential yield (PP) with the FAO Agroecological Zone method, penalized by the water deficit for each crop phase. Average EAs for corn, soybean and wheat crops were 54, 61 and 43\%, respectively. On the contrary of the majority of location, in Santa Rosa, São Borja and Veranópolis EA values were negative for soybean crop. The main factors that contributed to the increase of EA, in the majority of the locations, were: change on the soil use and fertility; use of agricultural machinery; prices paid for commodities; investments in research and development; climatic risk zoning; and plant breeding.
\end{abstract}

Key words: technological advances, potential yield, achievable yield and actual yield.

\section{INTRODUÇÃO}

O conceito de eficiência agrícola (EA) é utilizado como indicador do nível de desenvolvimento agrícola regional, sendo a relação entre a energia efetivamente utilizada e a que foi fornecida ao sistema (MARIN et al., 2008). Esses mesmos autores adotaram esse conceito, considerando como EA a razão entre a produtividade observada a campo e a atingível (PA), estimada por modelos de simulação de crescimento de plantas.

'Departamento de Engenharia de Biossistemas, Escola Superior de Agricultura "Luiz de Queiroz" (ESALQ), Universidade de São

Paulo (USP), CP 09, 13418-900, Piracicaba, SP, Brasil. E-mail: r.battisti@ @otmail.com. *Autor para correspondência.

IIDepartamento de Fitotecnia, Universidade Federal de Santa Maria (UFSM), Santa Maria, RS, Brasil. 
APAé aquela obtida a partir da penalização da produtividade potencial ( $\mathrm{PPf}$ ) pelo déficit hídrico que ocorre em cada fase de desenvolvimento da cultura. A PPf é aquela obtida em função das características da cultura e as variáveis do ambiente, como radiação solar, temperatura e fotoperíodo (PEREIRA et al., 2002). De acordo com PEREIRA et al. (2002), o déficit hídrico ocorre quando a evapotranspiração real é menor que a evapotranspiração da cultura. Quanto maior for o déficit hídrico, maior será o efeito deste sobre a redução da produtividade. Esse efeito do déficit hídrico sobre a produtividade é expresso por meio de um fator de sensibilidade ao déficit hídrico (Ky), o qual é função do estádio de desenvolvimento da cultura.

A produtividade real (PR), por outro lado, é aquela ocorrida a campo, normalmente menor que a PA, por considerar, além dos efeitos das condições meteorológicas, os efeitos do nível de manejo da cultura, o que contempla a correção química e fertilização do solo, as práticas de conservação do solo, o controle de pragas e doenças, o controle da matocompetição, entre outras. Para o milho, BORTOLINI et al. (2000) destacam que a baixa produtividade dessa cultura é devido a vários fatores, com destaque para a limitação de nitrogênio e para os danos causados pela lagarta do cartucho. Para as culturas da soja e do trigo, as doenças fúngicas, como a ferrugem asiática da soja e a giberela do trigo, também representam fatores de manejo que limitam o aumento da produtividade dessas culturas.

Diante da importância das culturas da soja, do milho e do trigo para o estado do Rio Grande do Sul (RS), e considerando-se que a EA depende da evolução tecnológica, o objetivo deste trabalho foi avaliar as EAs dessas três culturas em dez localidades do estado, analisando sua evolução entre os anos de 1980 e 2008 e identificando os principais fatores que as vêm condicionando.

\section{MATERIAL E MÉTODOS}

Para a estimativa da EA, foram utilizados os valores de produtividade real (PR) e atingível (PA) entre 1980 e 2008 para as culturas da soja, do milho e do trigo para as seguintes localidades: Encruzilhada do Sul

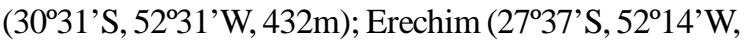

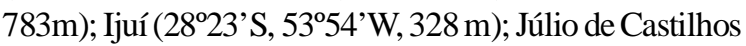
( $29^{\circ} 13^{\prime} \mathrm{S}, 53^{\circ} 40^{\prime} \mathrm{W}, 513 \mathrm{~m}$ ); Passo Fundo ( $28^{\circ} 15^{\prime} \mathrm{S}$, $52^{\circ} 24^{\prime} \mathrm{W}, 690 \mathrm{~m}$ ); Santa Maria (29⒋ 'S, 53⒊' 'W, 113m); Santa Rosa (27 $52^{\prime}$ 'S, $\left.54^{\circ} 28^{\prime} \mathrm{W}, 227 \mathrm{~m}\right)$; São Borja

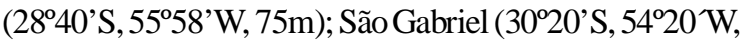

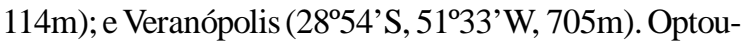
se por essas localidades em função da disponibilidade de dados climáticos e por contemplarem três regiões do estado (planalto, depressão central e escudo sulrio-grandense), as quais englobam as principais regiões produtoras de soja, milho e trigo do RS.

A PR foi obtida da base de dados SIDRA do Instituto Brasileiro de Geografia e Estatística (IBGE), considerando-se o valor correspondente à média anual por localidade. A PA foi estimada pela penalização da produtividade potencial (PPf) pelo déficit hídrico na escala decêndial, utilizando-se a equação a seguir: $\mathrm{PA}=[1-\mathrm{ky}(1-\mathrm{ETr} / \mathrm{ETc})] * \mathrm{PPf}$, em que:kyé ocoeficiente de sensibilidade ao déficit hídrico, obtido em DOORENBOS \& KASSAM (1994), de 0,4; 1,5 e 0,2 para o milho, 0,$2 ; 0,8$ e 1 para a soja e de 0,$2 ; 0,6$ e 0,0 para o trigo, correspondente às fases vegetativa, floração e maturação, enquanto que ETr e ETc são, respectivamente, a evapotranspiração real e da cultura.

Para a obtenção da ETc, estimou-se a evapotranspiração de referência pelo método de Camargo descrito em PEREIRA et al. (1997), a qual foi então multiplicada pelo coeficiente de cultura $(\mathrm{kc})$. Para o milho, utilizou-se kc médio de 0,7 para a fase vegetativa, 1,2 para a floração e 0,6 para a maturação (EMBRAPA, 2009); para a soja, foi utilizado kc de 0,5 para fase vegetativa e maturação, e de 1,15 para a floração (FARIAS et al., 2001); para trigo, de 0,75 para a fase vegetativa, 0,85 para a floração e 0,65 para a maturação (CUNHA et al., 2001b). Quanto à ETr, esta foi estimada por meio do balanço hídrico da cultura, de acordo com o método de THORNTHWAITE \& MATHER (1955), em escala decêndial, com capacidade de água disponível no solo (CAD) de 50mm (CUNHA et al., 2001a).

A PPf foi obtida pelo método da Zona Agroecológica, proposto por DOORENBOS \& KASSAM (1994), o qual utiliza como variáveis de entrada: o índice de área foliar máximo obtido pela cultura, correspondente a 5 para milho (STRIEDER et al., 2008), 4,5 para a soja (RODRIGUES et al., 2001) e 4 para o trigo (MOREIRA et al., 1999); parte colhida da matéria seca total produzida, de 0,5 para a cultura do milho (BONNECARRÈRE et al., 2007), 0,35 e 0,4, respectivamente, para as culturas da soja e do trigo (DOORENBOS \& KASSAM, 1994); duração de ciclo de produção, fixado em 150 dias para o milho (MALUF et al., 2001), 140 dias para a soja (CUNHA et al., 2001b) e 130 dias para o trigo (OSÓRIO \& WENDT, 1995); umidade residual na parte colhida de 11, 13 e 13,5\% (DOORENBOS \& KASSAM, 1994), respectivamente, para as culturas do milho, da soja e do trigo. Além das variáveis relativas às características das culturas, utilizou-se a temperatura do ar, a insolação, a irradiância solar global extraterrestre e o fotoperíodo, todos em 
escala decêndial, para a estimativa da produtividade bruta padrão (PPBp). A seguir, é apresentada a equação geral do método de Zona Agroecológica para estimativa de $\mathrm{PP}_{\mathrm{f}}$ :

$\mathrm{PP}_{\mathrm{f}}=\left(\mathrm{PPB}_{\mathrm{P}} * \mathrm{C}_{\mathrm{IAF}} * \mathrm{C}_{\mathrm{R}} * \mathrm{C}_{\mathrm{C}} * \mathrm{ND}\right) /(1-0,01 * \mathrm{U} \%)$, em que $\mathrm{PPB}_{\mathrm{p}}$ é a produtividade bruta de matéria seca para uma cultura padrão; $\mathrm{C}_{\mathrm{IAF}}$ correção do índice de área foliar máxima; $\mathrm{C}_{\mathrm{R}}$ correção da taxa de respiração; $\mathrm{C}_{\mathrm{C}}$ correção do índice de colheita; U\% umidade residual na parte colhida e ND é o número de dias do ciclo da cultura. Detalhes das determinações de $\mathrm{C}_{\mathrm{IAF}}$ e $\mathrm{C}_{\mathrm{R}}$ podem ser obtidos em PEREIRA et al. (2002).

Para as estimativa de PA, simularam-se, a cada 10 dias, as datas de semeadura recomendadas pelo zoneamento agrícola, de 21/07 a 11/01 para a cultura do milho (MALUF et al., 2001), de 01/10 a 21/12 para a cultura da soja (CUNHA et al., 2001a) e de 01/05 a 21/07 para a cultura do trigo(CUNHA et al., 2001b). Os valores de PA de cada época de semeadura foram usados no cálculo da PA média de cada localidade, sendo esse valor empregado então para a determinação da EA, juntamente com os dados de PR do IBGE:

$\mathrm{EA}=(\mathrm{PR} / \mathrm{PA}) * 100$

Com a obtenção da EA para cada ano, compôs-se o valor médio da série para o RS e para as localidades avaliadas, além da inclinação da reta entre 1980 e 2008 com uso de análise de regressão linear. Após a obtenção dos resultados, buscou-se na literatura identificar os principais fatores associados à evolução tecnológica das culturas do milho, da soja e do trigo.

\section{RESULTADOS E DISCUSSÕES}

As PPfs para as culturas da soja, do trigo e do milho foram, em média, para a série em estudo, de $6.394,4.320$ e $12.032 \mathrm{~kg} \mathrm{ha}^{-1}$, respectivamente. Os valores anuais de PPf são apresentados na figura 1 , na qual pode-se observar que a inclinação da reta, representada pelo valor de "b", é menor do que para a PA e a PR, demonstrando valores constantes ao longo da série, pela baixa dispersão dos pontos. Para a PA, pode-se observar uma maior dispersão em relação à reta, devido ao fato de que essa produtividade é resultante da penalização da PPf pelo déficit hídrico, e que a chuva é uma das variáveis meteorológicas de maior variação interanual. Quanto aos valores médios de PA ao longo da série histórica, a cultura do trigo é a que teve a menor perda de produtividade, devido ao déficit hídrico, já que as retas de PPf e PA estão muito próximas (Figura 1B). O valor médio de PA do trigo foi de $3.802 \mathrm{~kg} \mathrm{ha}^{-1}$. Para as culturas da soja e do milho, os valores médios da PA foram respectivamente de $2.797 \mathrm{e} 5.083 \mathrm{~kg} \mathrm{ha}^{-1}$.
Ainda analisando a figura 1, pode-se observar que a linha de tendência de PR foi a que apresentou a maior inclinação, quando comparada às linhas da PPf e PA. Os valores de "b" obtidos foram $17,92,42,96$ e 77,58 para as culturas da soja, do trigo e do milho, com valores médios de PR de 1.639, 1.452 e $2.474 \mathrm{~kg} \mathrm{ha}^{-1}$ para o estado do RS. Assim, quanto maior a distância das linhas de tendência de PR e PA, menor é a EA.

Na tabela 1, são apresentados os resultados da linha de tendência da EA entre os anos de 1980 e 2008 e o valor médio para as dez localidades avaliadas no estado do RS. Para as culturas do milho, da soja e do trigo, os locais que apresentaram maior aumento da EA foram Passo Fundo, Santa Maria e Encruzilhada do Sul, respectivamente. Enquanto que em Santa Rosa, São Borja e Veranópolis houve redução da EA para a cultura da soja. Esses resultados estão associados à qualidade do manejo das culturas nessas áreas. Onde a EA aumentou, pode-se dizer que houve uma melhoria no manejo das lavouras, com a adoção de cultivares mais produtivas, correção do solo e fertilização, uso do plantio direto, entre outras práticas. Por outro lado, nas localidades onde ela diminuiu, como no caso da soja em Santa Rosa, São Borja e Veranópolis, o manejo dessa cultura foi de menor qualidade devido a questões econômicas associadas ao maior risco climático nessas áreas, especialmente em Santa Rosa e São Borja, onde houve redução dos investimentos na cultura ao longo do período analisado, por questões de perdas de produtividade devido à alta frequência de veranicos. Já em Veranópolis, a redução da EA entre 1980 e 2008 esteve associada à migração das áreas de soja para frutíferas (IBGE, 2010).

No caso da cultura do milho, o valor do coeficiente angular (b) foi de 0,013 (Tabela 1), ou seja, um aumento médio de 1,3\% na EA por ano, acumulando $37,7 \%$ entre 1980 e 2008, destacando-se as localidades de Passo Fundo e Erechim, em que o aumento acumulado chegou a $63 \%$. Para a cultura da soja, o aumento anual da EA foi de $0,3 \%$, acumulando $8,7 \%$ no período, destacando-se a localidade de Santa Maria, onde o aumento acumulado de EA chegou a $40 \%$. Quanto à cultura do trigo, o aumento anual médio da EA foi de $0,9 \%$, acumulado de $26,1 \%$ no período, destacando-se a localidade de Encruzilhada do Sul, onde o aumento chegou a 43,5\%, enquanto que em Ijuí não passou de $11,6 \%$. Para a cultura do trigo, este ganho anual demonstra que, apesar do baixo retorno econômico dessa cultura no estado, ao longo do período, ainda houve um ganho de produtividade. $\mathrm{Na}$ cultura da soja, a EA variou entre 49 e $79 \%$ (Tabela 1), ou seja, a produtividade real foi de 49 a $79 \%$ da PA. 


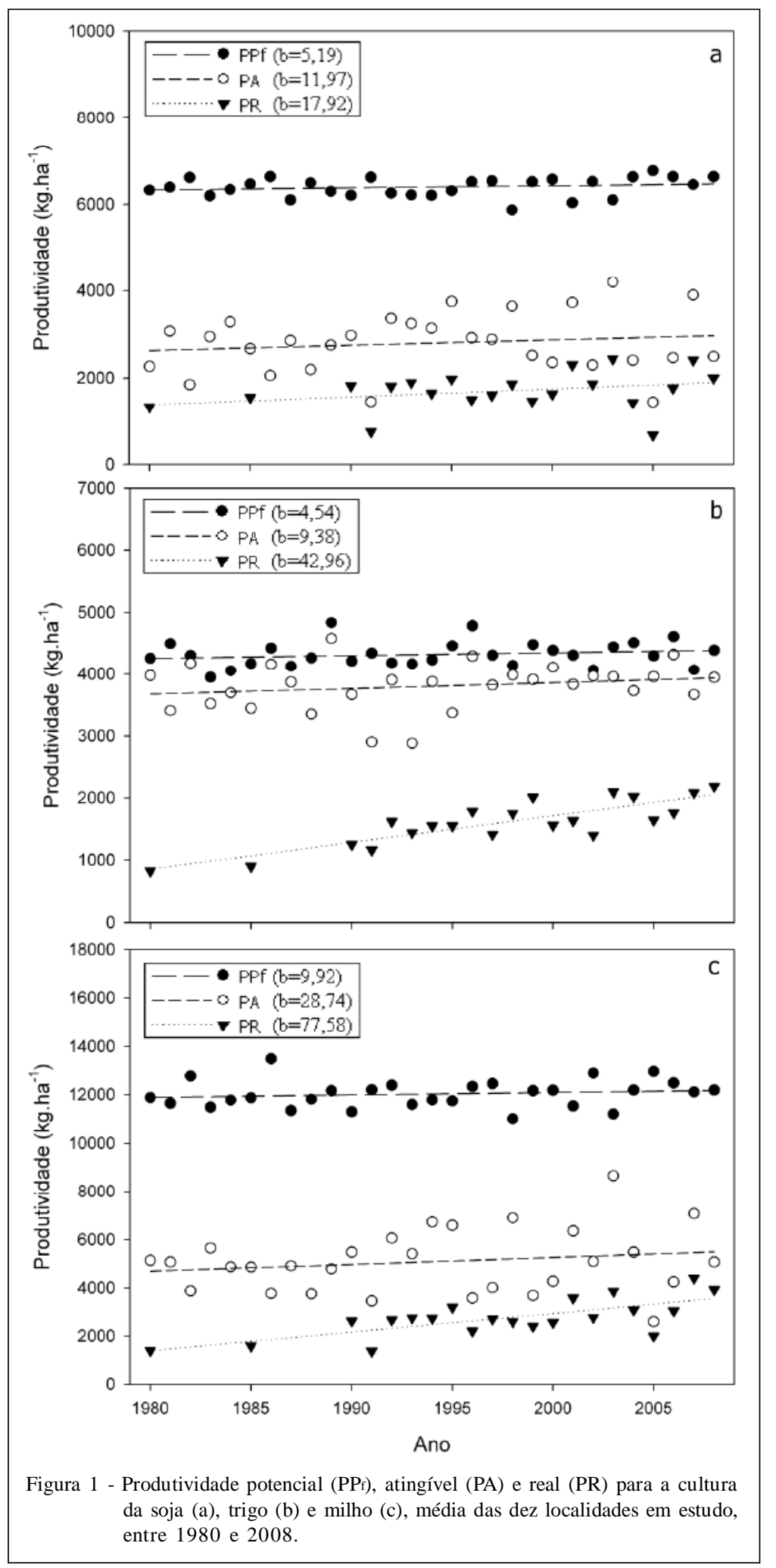

Ciência Rural, v.42, n.1, jan, 2012. 
Tabela 1 - Coeficiente angular (b) para evolução temporal da eficiência agrícola e eficiência agrícola média (EA), para as culturas do milho, soja e trigo nas dez localidades em estudo, no estado do Rio Grande do Sul.

\begin{tabular}{|c|c|c|c|c|c|c|c|}
\hline \multirow{2}{*}{ Local/Cultura } & \multicolumn{2}{|c|}{------------Milho------------ } & \multicolumn{2}{|c|}{------------Soja------------ } & \multicolumn{2}{|c|}{----------Trigo------------ } & \multirow{2}{*}{$\begin{array}{c}\text { EA Média } \\
(\%)\end{array}$} \\
\hline & $\mathrm{b}$ & $\mathrm{EA}(\%)$ & $\mathrm{b}$ & $\mathrm{EA}(\%)$ & $\mathrm{b}$ & $\mathrm{EA}(\%)$ & \\
\hline Encruzilhada do Sul & 0,010 & 39 & 0,008 & 62 & 0,015 & 41 & 47 \\
\hline Erechim & 0,022 & 57 & 0,010 & 54 & 0,010 & 43 & 52 \\
\hline Ijuí & 0,014 & 65 & 0,001 & 65 & 0,004 & 38 & 56 \\
\hline Julio de Castilhos & 0,013 & 59 & 0,003 & 65 & 0,010 & 44 & 56 \\
\hline Passo Fundo & 0,025 & 61 & 0,008 & 55 & 0,009 & 49 & 55 \\
\hline Santa Maria & 0,009 & 60 & 0,014 & 79 & 0,012 & 49 & 63 \\
\hline Santa Rosa & 0,011 & 53 & $-0,006$ & 55 & 0,008 & 38 & 49 \\
\hline São Borja & 0,006 & 39 & $-0,008$ & 49 & 0,005 & 41 & 43 \\
\hline São Gabriel & 0,002 & 47 & 0,006 & 74 & 0,009 & 46 & 56 \\
\hline Veranópolis & 0,017 & 58 & $-0,004$ & 57 & 0,014 & 36 & 50 \\
\hline Média & 0,013 & 54 & 0,003 & 61 & 0,009 & 43 & 53 \\
\hline
\end{tabular}

Para a cultura do milho, a EA foi inferior à da cultura da soja, para a maioria das localidades, com exceção de Erechim e Passo Fundo, resultado que pode estar associado às melhores condições físicas do solo da região, com maiores valores de capacidade de armazenamento de água no solo, beneficiando o investimento nesta cultura, que é mais sensível ao déficit hídrico do que a cultura da soja. Para o trigo, a EA variou de 36 a $49 \%$.

Avaliando a EA média por cultura, a soja apresentou valor de $61 \%$, enquanto que o milho obteve $54 \%$ e o trigo $43 \%$ (Tabela 1). Isso indica que o sistema de produção para as culturas da soja, do milho e do trigo tem eficiência moderada. Para todas as localidades, a EA média das três culturas foi de 43 a $63 \%$ (Tabela 1), destacando-se que a maioria das localidades apresentou EA maior que $50 \%$ na média das três culturas.

Na figura 2, é apresentada a EA para o estado do RS para as culturas do milho, soja e trigo entre 1980 e 2008, com o respectivo valor da inclinação da reta (b), demonstrando que houve um ganho de EA ao longo do período. Pode-se verificar que a cultura da soja apresentou a menor inclinação da reta $(b=0,003)$, enquanto que o milho teve o maior ganho para o período $(b=0,013)$. A cultura do trigo sempre esteve com a EA abaixo das demais culturas, com inclinação da reta intermediária $(b=0,009)$.

Dentre os fatores que contribuíram para a elevação da EA, destacam-se a melhoria da fertilidade e uso do solo, mecanização agrícola, preços pagos pelas commodities, investimento em pesquisa e desenvolvimento, zoneamento de risco climático e melhoramento genético. MARIN et al. (2008) verificaram que o aumento da EA em cana-de-açúcar esteve associado às condições de manejo, os quais foram impulsionados pelo maior retorno econômico da cultura.

Para o uso e a fertilidade do solo, uma prática que melhora os atributos do solo é o sistema de plantio direto. Segundo NICOLODI et al. (2009), em 1980, a área com plantio direto no RS era menor que $5 \% \mathrm{e}$, em 2000 , passou a representar mais de $80 \%$ da área de cultivo. Além disso, destaca-se a parte química, como o pH, matéria orgânica e concentração de fósforo e potássio, que apresentam influência direta na produtividade. Segundo esses mesmos autores, a baixa produtividade no RS na década de 60 era causada, principalmente, pelo baixo teor de $\mathrm{Pe} \mathrm{K}$, alta acidez e manejo inadequado dos solos e das culturas.

Para o pH, NICOLODI et al. (2009) observaram inversão do percentual da área que apresentava valores menores que $5 \mathrm{em} \mathrm{1968,} \mathrm{para} \mathrm{um}$ maior percentual para o $\mathrm{pH}$ maior que 5,6 em 1999. Esta melhoria do pH, entre 1968 e 1999, esteve associada às políticas de disponibilidade facilitada de calcário para os produtores rurais. Ainda associado ao manejo de solo a médio/longo prazo, estes mesmos autores observaram aumento da área agrícola do estado com teores de matéria orgânica acima de 2,5\% e dos teores de fósforo e potássio disponível no solo de 1968 a 1999.

A mecanização agrícola também auxiliou na melhoria da EA, atuando em três pontos decisivos: semeadura, tratos culturais e a colheita. A partir de dados do IBGE (2010), observou-se que, entre 1970 e 2006, ocorreu um aumento substancial no número de colhedoras e tratores no RS, reduzindo assim a superfície de cultivo para cada unidade, auxiliando no aumento da EA.

Além de práticas de manejo, questões financeiras como o preço que o produtor rural recebe pelo que vem produzindo tem papel fundamental na 


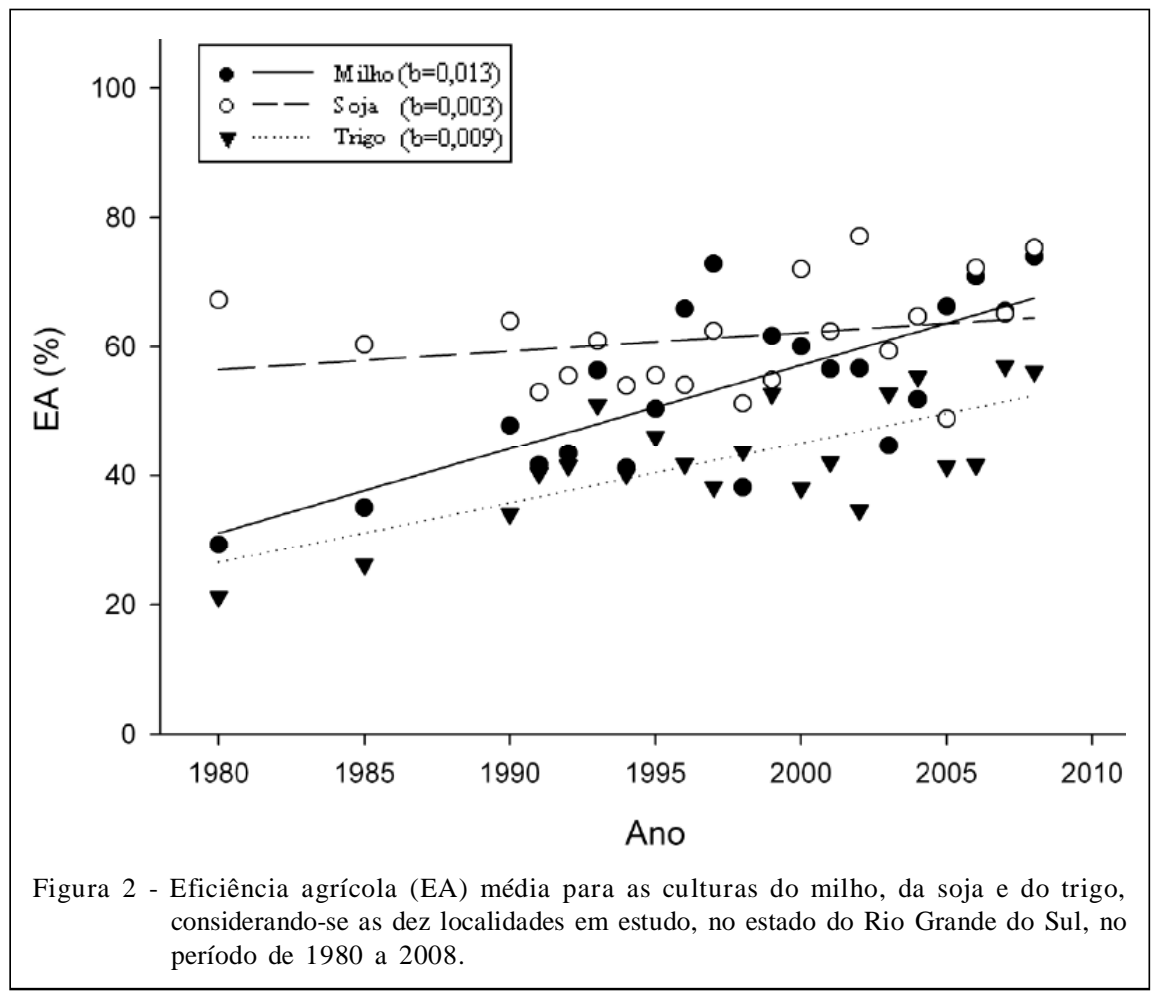

EA. SOUZA \& VIANA (2007) observaram redução dos preços históricos pagos pelo milho, soja e trigo e, segundo esses autores, a redução de preço está baseada na lei clássica de oferta e procura e que com a redução de preços tornou-se necessário que o produtor rural aumentasse a EA para que fosse viável economicamente manter a atividade rural.

O desenvolvimento de novas tecnologias, sejam elas produtos ou técnicas de cultivos, sempre auxiliam no aumento da produtividade e, consequentemente, da EA. No Brasil, a EMBRAPA (Empresa Brasileira de Pesquisa Agropecuária) tem papel fundamental no desenvolvimento agropecuário. Assim, o total de investimento recebido por este órgão de pesquisa pode representar o desenvolvimento tecnológico para todo o país. Segundo GONÇALVES (2006), os valores de investimento feitos na EMBRAPA pelo governo federal foram de $\mathrm{R} \$ 950$ milhões em 2005 , quase cinco vezes superior ao feito em 1975. Associado à pesquisa, o zoneamento agrícola de risco climático buscou aumentar a produtividade no âmbito nacional, delimitando as melhores áreas e épocas de cultivo, a partir do cruzamento de dados de solo, clima e exigências das culturas, fazendo com que as fases mais críticas tivessem menor probabilidade de coincidirem com adversidades climáticas (INPE, 2010), tornandose uma alternativa de escape ao déficit hídrico (CUNHA et al., 2001a).
Dentro da melhoria de produtividade, a seleção de plantas superiores é uma importante ferramenta. Nesse contexto, o melhoramento genético por meio de técnicas clássicas ou de biotecnologia auxilia no aumento da EA. Segundo FERREIRA (2006), o ganho genético anual atual no Brasil é menor que $1 \%$, ao passo que na década de 60 esse ganho era na ordem de $3 \%$. Como o ganho genético vem se reduzindo gradativamente, o aumento da EA devido ao melhor manejo das culturas é uma das principais alternativas para o aumento das produtividades no futuro.

\section{CONCLUSÃO}

Observou-se que, durante o período avaliado, entre 1980 e 2008, ocorreu um ganho de EA para as culturas da soja, do milho e do trigo na média das dez localidades. Apenas em Santa Rosa, São Borja e Veranópolis, houve redução da EA para a soja. Dentre os fatores que contribuíram para o aumento da EA, destacam-se a melhoria da fertilidade e uso do solo, mecanização agrícola, preço pago às commodities, investimento em pesquisa e desenvolvimento, zoneamento agrícola de risco climático e melhoramento genético. 


\section{REFERÊNCIAS}

BONNECARRÈRE, R.A.G. et al. Estimativa das produtividades potencial e deplecionada da cultura de milho no Estado do Rio Grande do Sul em função das condições climáticas. Rev Bras de Agrometeorologia, v.15, n.3, p.280-288, 2007.

BORTOLINI, C.G. et al. Sistemas consorciados de aveia preta e ervilha comum como cobertura de solo e seus efeitos na cultura do milho em sucessão. Rev Bras Ci Solo, v.24, p.897903, 2000.

CUNHA, G.R. da, et al. Zoneamento agrícola e época de semeadura para a soja no Rio Grande do Sul. Rev Bras de Agrometeorologia, v.9, n.3, p.446-459, 2001 a.

CUNHA, G.R. da, et al. Zoneamento agrícola e época de semeadura para trigo no Brasil. Rev Bras de Agrometeorologia, v.9, n.3, p.400-414, 2001b.

DOORENBOS, J.; KASSAM, A.M. Efeito da água no rendimento das culturas. Campina Grande: FAO, 1994. (Estudos FAO, Irrigação e Drenagem 33).

EMBRAPA. Sistemas de produção - Milho. 5.ed. Versão Eletrônica, 2009. Acesso em: 08 set. 2010. Online. Disponível em: <http://sistemasdeproducao.cnptia.embrapa.br/FontesHTML/ Milho/CultivodoMilho_5ed/index.htm>.

FARIAS, J.R.B. et al. Caracterização de risco de déficit hídrico nas regiões produtoras de soja no Brasil. Rev Bras de Agrometeorologia, v.9, n.3, p.415-421, 2001.

FERREIRA, M.E. Aporte biotecnológico ao melhoramento genético de plantas. Brasília-DF, Brasil, 2006. Acesso em: 12 nov. 2010. Online. Disponível em: <http://www.cenargen.embrapa.br/ pre-melhoramento/Por/prog_por.htm $>$.

GONÇALVES, J.S. Agropecuária paulista e brasileira: crescimento do produto e investimento em P\&D. Análises e indicadores do agronegócio, v.1, n.10, out. p.9, 2006.

IBGE (Instituto Brasileiro de Geografia e Estatística). Produtividade agrícola municipal. Acesso em: 22 ago. 2010. Online. Disponível em: <http://www.sidra.ibge.gov.br/>.

INPE. Importância do zoneamento agrícola e épocas de plantio de cada município. Acesso em: 11 nov. 2010. Online. Disponível em: <http://agricultura.cptec.inpe.br/>.

MALUF, J.R.T. et al. Zoneamento de risco climático para a cultura de milho no Rio Grande do Sul. Rev Bras de Agrometeorologia, v.9, n.3, p.460-467, 2001.
MARIN, F.R. et al. Sugarcane crop efficiency in two growing seasons in São Paulo State, Brazil. Pesq Agropec Bras, v.43, n.11, p.1449-1455, 2008. Disponível em: <http://www.scielo.br/ scielo.php? script $=$ sci_art text \& pid = S 0100 204X2008001100002\&lng=pt\&nrm=iso >. Acesso em: 15 out. 2010. doi: 10.1590/S0100-204X2008001100002.

MOREIRA, M.A. et al. Eficiência do uso da radiação e índice de colheita em trigo submetido a estresse hídrico em diferentes estádios de desenvolvimento. Sci agric, v.56, n.3, p.597-603, 1999. Disponível em: <http://www.scielo.br/scielo.php?script=sci_arttext\&pid=S0103$90161999000300012 \& \operatorname{lng}=$ pt\&nrm=iso $>$. Acesso em: 28 set. 2010. doi: 10.1590/S0103-90161999000300012.

NICOLODI, M. et al. Evolução da fertilidade do solo do Planalto do Rio Grande do Sul nas últimas quatro décadas. Rev Plantio Direto, Ed. 111, p.10-16, 2009.

OSÓRIO, E.A.; WENDT, W. Duração do período de formação do grão em trigo. Sci agric, v.52, n.2, p.395-398, 1995. Disponível em: <http://www.scielo.br/scielo.php?script=sc i_arttext\&pid=S010390161995000200031\&lng=pt\&nrm=iso>. Acesso em: 20 set. 2010. doi: 10.1590/S0103-90161995000200031.

PEREIRA, A.R. et al. Agrometeorologia: fundamentos e aplicações práticas. Guaíba: Agropecuária, 2002. 478p.

PEREIRA, A.R. et al. Evapo(transpi)ração. Piracicaba: FEALQ, 1997. 183p.

RODRIGUES, O. et al. Resposta quantitativa do florescimento da soja à temperatura e ao fotoperíodo. Pesq agropec Bras, v.36, n.3, p.431-437, 2001. Disponível em: <http:// www.scielo.br/scielo.php? script $=$ sci_arttext\&pid=S0100 $204 \mathrm{X} 2001000300006 \& \operatorname{lng}=$ pt\&nrm=iso $>$. Acesso em: 08 set. 2010. doi: 10.1590/S0100-204X2001000300006.

SOUZA, R.S. de; VIANA, J.G.A. Tendência histórica de preços pagos ao produtor na agricultura de grãos do Rio Grande do Sul, Brasil. Ciênc Rural, v.37, n.4, p.1128-1133, 2007. Disponível em: <http:/ /www.scielo.br/scielo.php?script $=$ sci_arttext\&pid $=$ S0 103 84782007000400034\&lng=pt\&nrm=iso>. Acesso em: 13 nov. 2010. doi: $0.1590 / \mathrm{S} 0103-84782007000400034$.

STRIEDER, M.L. et al. Características de dossel e rendimento de milho em diferentes espaçamentos e sistemas de manejo. Pesq agropec bras, v.43, n.3, p.309-317, 2008. Disponível em: <http:/ $/$ www.scielo.br/scielo.php? script $=$ sci_arttext\&pid=S0100204X200800 0300004\&lng=pt\&nrm=iso >. Acesso em: 08 out. 2010. doi: 10.1590/S0100-204X2008000300004.

THORNTHWAITE, C.W.; MATHER, J.R. The water balance. Publications in climatology. New Jersey: Drexel Institute of technology, 1955. $104 \mathrm{p}$. 